\title{
Space Research on Organs and Tissues
}

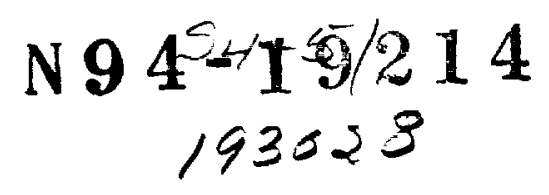

Marc E. Tischler

Department of Biochemistry, University of Arizona, Tucson, AZ 85724

EMILY MOREY-HoLTON

NASA, Ames Research Center, Moffett Field, CA 94035

\section{ABSTRACT}

Studies in space on various physiological systems have and will continue to provide valuable information on how they adapt to reduced gravitational conditions, and how living in a $1 \mathrm{~g}$ (gravity) environment has guided their development. Muscle and bone are the most notable tissues that respond to unweighting caused by lack of gravity. The function of specific muscles and bones relates directly to mechanical loading, so that removal of "normal forces" in space, or in bedridden patients, causes dramatic loss of tissue mass. The cardiovascular system is also markedly affected by reduced gravity. Adaptation includes decreased blood flow to the lower extremities, thus decreasing the heart output requirement. Return to $1 \mathrm{~g}$ is associated with a period of reconditioning due to the deconditioning that occurs in space. Changes in the cardiovascular system are also related to responses of the kidney and certain endocrine (hormone-producing) organs. Changes in respiratory function may also occur, suggesting an effect on the lungs, though this adaptation is poorly understood. The neurovestibular system, including the brain and organs of the inner ear, must adapt to the disorientation caused by lack of gravity. Preliminary findings have been reported for liver. Additionally, endocrine organs responsible for release of hormones such as insulin, growth hormone, glucocorticoids, and thyroid hormone may respond to spaceflight.

\section{INTRODUCTION}

Microgravity, the decreased effectiveness of gravity, produces profound effects on the body's biochemistry and physiology. Systems affected include muscle, bone, cardiovascular, pulmonary, neurovestibular, liver, and endocrine. For humans to live and work in space, it is essential that we identify the precise consequences of exposure to microgravity and then develop appropriate countermeasures, if necessary. Furthermore, understanding how animals and humans adapt to microgravity will provide a clearer picture of how gravity has influenced the development of these systems under $1 \mathrm{~g}$ conditions. The key word here is "adaptation." Microgravity should not be considered a pathological state. Organs and tissues are simply adapting to the new physi- ological state or environment, just as one's body must adapt to a change from functioning at sea level to performing at high altitude. For instance, decreased oxygen pressure at high altitude requires an increase in the number of red cells in the blood for transporting vital oxygen to peripheral tissues and organs. Thus the cardiovascular system is exquisitely sensitive to changes in the physiological environment of the organism.

Our precise knowledge of the effects of microgravity on humans and animals is still limited for several reasons: (1) the sample size is too small to make many generalizations; (2) the capabilities for scientific studies, including long-term flights, is limited; (3) astronauts have used a variety of countermeasures, thus obscuring effects of weightlessness; (4) there is considerable variation in the types of missions flown including duration, numbers of crewmembers, and goals of the mission, including the planned animal studies; (5) a difficulty with interpreting flight data is the tremendous variability among human subjects, which may be related to their physical status at the onset of flight; and (6) our space program faced a major setback because of the Challenger disaster in 1986. Therefore, most studies have concerned animals using primarily Earth-bound models to mimic the potential effects of unloading, the removal of the weight or mass supported by muscle or bone using artificial support or microgravity. These model systems are characterized by hypodynamia, the deprivation of normal weight-bearing function, and hypokinesia, the deprivation of normal locomotive function, both of which are common to space travel. It has also been possible using these models to mimic the body fluid shifts expected in spaceflight, as a means of testing alterations of the cardiovascular system. However, some alterations of organs and tissues caused by microgravity are not reproducible in Earth-bound animal or human models. Thus, space research on organs and tissues is essential both for validating the Earth-bound models used in laboratories, and for studying the adaptations to weightlessness that cannot be mimicked on Earth. 


\section{MUSCLE METABOLISM AND PHYSIOLOGY}

\section{Background}

Skeletal muscle, which is striated muscle, is composed of bundles of fibers that can shorten or lengthen as necessary. These muscles function in support, locomotion, and work. Additionally, protein in these muscles provides fuel for the body during prolonged food deprivation or following severe injury. During work production, the total muscle force, which reflects the actual power exerted in producing motion or overcoming opposition, depends on the sum of all fibers. Strength, which is defined as the inherent capacity of the muscle, is related to the cross-sectional area of these fibers. Hence, a decrease in fiber size and/or number will affect the work capacity of a muscle. Energy for muscle function is derived largely from glycogen and fats. Glycogen is stored in muscle and is readily available. Therefore, glycogen is used when work begins, followed later by fats from the blood.

Muscles that assist us in standing consume about $15 \%$ more energy than when the body is in a supine position. Not all muscles depend on gravity for their function, and the role of these muscles does not change in microgravity. Muscles that depend on gravily are termed "antigravity" and are located generally in the legs, back, face, and neck. These muscles have different types of fibers than nonpostural muscles. In the absence of an antigravity role, muscles may revert to alternate types of fibers.

\section{Spaceflight and Simulation Studies}

Measurements from various missions show space travel causes atrophy (wasting) of certain muscles. About $15 \%$ of weight loss is due to loss of muscle mass. Muscles whose functions do not depend on bearing weight (e.g., arm muscles) fail to lose mass. Therefore, a large loss by a select group of muscles must account for the bulk of the responses. A recent spaceflight study (STS-48, September 1991) of muscle in young rats showed that some weight-bearing muscles simply grow slower, rather than atrophy. Thus muscle response is complicated by preexisting physiological status.

In humans, the lower extremities lose volume, a third of which is muscle atrophy, but the arms maintain their volume. Indeed, in microgravity, the function of the arms changes to one of locomotion and stability. These results suggest that the loss of muscle mass, especially in the legs, is due to mechanical factors.

Muscle atrophy is associated with breakdown of muscle protein, revealed by the excretion of break- down products, various nitrogen-containing compounds, in the urine. Because nitrogen intake is not increased, the accelerated excretion must result from an excess of the breakdown of muscle proteins over their formation.

Wasting produces physiological changes in muscle. Generally, these adaptations are not a problem until the muscles must function again under the influence of gravity. With extended periods in space, the problems become more severe. Cosmonauts exposed to eight months or more of microgravity generally have difficulty in walking and in maintaining proper posture. Microgravity causes a decrease in muscle tone, work capacity, efficiency, and strength, with an increase in fatigability. Recovery from the effects of space travel varies with the duration of the mission. Muscle strength may require from several days to several weeks to return to normal (i.e., $1 \mathrm{~g}$ ).

Bedrest studies have been used to test effects of unloading on the musculoskeletal system. Bedrest is not a true simulation because of the ever-present influence of gravity; however, results from such studies are remarkably similar to what we have learned in space. With bedrest, and as is well-known from bedridden patients, there is loss of leg mass and volume, as well as a decrease in the cross-sectional area of muscle fibers and a change in fiber type of the antigravity muscles. Bedrest studies have provided an opportunity to compare the effects of muscle unloading in men and women. Still, such models are limited by being conducted under the influence of $1 \mathrm{~g}$.

Many of the advances in our knowledge of the biochemistry of muscle atrophy have come about by using animal models, as mentioned in the Introduction. Studies on Earth have used hindlimb unweighting to mimic the effects of microgravity on muscle and bone. Limited studies have also been conducted on rats subjected to microgravity. In any event, animals represent a major part of research on the effects of hypokinesia and hypodynamia.

Associated with muscle atrophy are significant physiological changes in the affected muscles, including increased fatigability; decreased strength, elasticity, and force; smaller fiber cross-sectional areas; and change in fiber type of antigravity muscles. Therefore, physiological changes in unloaded rat muscle parallel at least some of those found for unloaded human muscle.

Biochemical studies on unloaded rat muscle have considered the adaptation of carbohydrate, amino acid, and protein metabolism to this intervention. With decreased muscle use, there is a buildup of glycogen, presumably because of decreased utilization of this fuel. The unloaded muscle may respond more to insulin, which protects the muscle to a small extent against loss of protein, accounting for the prolonged period of slower muscle loss following the 
initial rapid atrophy. Finally, measurements of protein formation and protein breakdown in the unloaded muscle using the animal model suggest that abnormalities in both processes may contribute to the muscle wasting.

\section{BONE AND MINERAL METABOLISM}

\section{Background}

Bone provides mechanical support to the body and plays an important role in the regulation of body calcium and phosphorus. While it is clear that biomechanical activity controls the interaction of bone and calcium, the mechanisms related to bone growth, mineralization, and maintenance are still poorly understood. Bone mass is in part maintained by loading the bone under the influence of gravity. Muscle tension on bone is also of importance. Bone, like muscle, adapts to limb immobilization and/or unloading. In adult humans, removing the load leads to osteopenia (bone "wasting"), which eventually can cause decreased bone strength and a reduced ability of fractures to heal. The primary bone mineral is calcium phosphate. The bone matrix is primarily protein, of which $95 \%$ is collagen. Collagen is unique in that it contains a large proportion of the amino acid hydroxyproline.

Our concepts of the response of the skeletal system to gravitational loading are changing as more information becomes available. During spaceflight, bone responds to an environment where movement of the body and the loads imposed during movement are different from those on Earth. The response to these changes are adaptive, not pathological. The adaptive response to spaceflight involves the entire skeleton, and different parts of the skeleton respond differently. Skeletal adaptation is determined by loading history which, in turn, is a function of exercise, body mass (weight), muscle forces, and fluid pressure and distribution. The ability to adjust to changes in mechanical loading is dependent not only on loading history, but also on normal hormone levels and nutritional intake.

\section{Spaceflight and Simulation Studies}

Our current theory suggests that spaceflight, concomitant with the near lack of body weight and the changes in body movements, changes muscle mass. These adjustments, along with fluid shifts, which probably modify blood flow to tissues, cause changes in mechanical loading. The skeleton somehow senses the altered load and adapts to its altered function. The adaptation varies from site to site within the skeleton, depending on the change in the loading history. The head and possibly the arms may accumulate mineral while the legs and the trunk lose mass. Bones in which maintenance of mineral is gravity-dependent (i.e., due to ground reaction forces) lose mineral, and individuals who are exercisers will probably lose mass in these bones more rapidly than sedentary individuals. Bone loss will occur generally coincident with, though on a slower time scale than, muscle atrophy and decreased muscle strength. These skeletal changes alter the calcium fluxes in and out of bone. The net result of this process is an increase in serum calcium, which initiates a hormone cascade. The increase in serum calcium is usually attributed to increased bone resorption. However, the response is more likely a combination of decreased mineralization with less calcium going into bone and site-specific increases in resorption with increases in calcium fluxes from the bone.

The skeleton adapts to spaceflight as long as diet and endocrine (hormonal) milieu are adequate. The adaptation is a normal physiological process and not a disease state. The result of the adaptation is a change in bone mass with altered architecture and composition. The altered architecture may be reflective of the functional changes of bone and may contribute to the changes in bone strength. However, this adaptation could impair the return to a $1 \mathrm{~g}$ environment. The role of bone in mineral homeostasis probably does not change. Spaceflight may be a unique environment to study perturbation of the mineral reservoir independent of loading effects.

The term "bone loss" has been used to describe spaceflight skeletal adaptation and is useful in conveying a site-specific response that triggers the systemic calcium-endocrine response, but the phrase is not appropriate for describing the entire skeleton. We suggest that "bone or skeletal adaptation process" more accurately describes the skeletal response to spaceflight.

Various measurements have been used to estimate bone adaptation. Increases in urine and fecal calcium provide one form of evaluation, though such measurements are affected by the factors described above. Measurements of bone density have been done primarily on the heel bone because of the tremendous load and stress placed on this bone under the influence of gravity. The density diminishes in proportion to the duration of the mission, though a large variability implies that other factors are of importance. For instance, the extent of adaptation may depend on the initial turnover rate of mineral content for each crewmember. Thus, prior physiological condition is an important determinant in the adaptation process.

Much of our understanding of the mechanisms of bone adaptation with unloading have come from 
bedrest simulations. Such studies showed a loss of minerals similar to that observed during spaceflight, especially in weight-bearing bones. No decrease in density was observed in bones of the upper extremities. While bedrest unloading does not precisely mimic microgravity unloading, simulation studies have allowed prediction of the rate and extent of mineral loss by certain bones.

Animal studies have been conducted in space or using unloading models, such as the couched monkey and the hindlimb-unweighted rat. Monkeys, like humans, show decreased bone mineral and evidence of increased bone resorption. In rats, however, the primary reason for decreased bone mass is a reduced rate of formation without significant resorption. This decreased formation in turn leads to slower growth, demineralization, and decreased bending strength. The different response of bone in rats than in humans or monkeys could be due to a different type of bone structure or to differences in limb motion and loading, or because adult rats show constant bone growth, unlike in adult humans.

Animal models are critical for answering specific questions about the biochemical and physiological adaptation of bone to unloading. Because the rat model does not mimic the response of human bone, it will be important to use other animal models to study the mechanisms of skeletal and calcium changes in space. Still, flight data are critical for validating these models. It is also essential to develop noninvasive analytical methods to facilitate the study of bone loss.

\section{CARDIOVASCULAR AND PULMONARY}

\section{Background}

The cardiovascular system transports and distributes essential substances (e.g., oxygen and nutrients) to the tissues and removes metabolic by-products. In addition, it contributes to the regulation of body temperature, to hormonal communication within the body, and to the exchange of materials, via the lungs, kidneys, and skin, with the external environment. The cardiovascular system includes the heart, blood, and blood vessels. Blood transports the essential substances described above and is comprised of plasma (the fluid component) and cells. The vessels (vasculature) include arteries, veins, and capillaries. The heart and the vasculature are divided into the systemic (body) and pulmonary (lungs) circulations. The amount of blood the heart pumps (cardiac output), the pressure exerted on the vessel walls (blood pressure), and other aspects of cardiovascular function are exquisitely controlled. Local blood flow is influenced by a variety of factors including the concentrations of oxygen, carbon dioxide, $\mathrm{pH}$, and metabolic compounds.

\section{Cardiovascular Adaptation to Spaceflight}

Under gravitational influence upon standing, blood accumulates in the lower extremities. This pooling increases pressure in vessels below the heart and decreases pressure above the heart. To ensure adequate blood flow to all areas of the body, especially the brain, mechanisms exist to adjust blood flow relative to gravity. Adaptations that occur in microgravity can help us to better understand the normal influences of gravity on Earth. Microgravity alters fluid gradients within the cardiovascular system such that fluid redistributes from the lower extremities to the head, neck, and torso. This redistribution of blood promotes diuresis (fluid loss through urine), which occurs primarily during the first day and continues for up to four days. Diuresis is primarily controlled by a decrease of antidiuretic hormone (ADH). ADH release from the posterior pituitary gland is regulated by a nerve signal from the right atria of the heart. To maintain normal plasma osmotic pressure, sodium (electrolyte) loss must be commensurate with fluid loss.

Plasma electrolytes are regulated by the reninangiotensin-aldosterone hormone cascade. Weightlessness decreases fluid pressure and nerve signals to the kidney, thus reducing renin release. Consequently, angiotensin formation and aldosterone release are decreased due to less renin. Aldosterone enhances sodium reabsorption in the kidney, such that aldosterone decrease can lead to a marked sodium loss.

Despite the limited inflight experimentation, it is clear that the cardiovascular system undergoes marked physiological alterations in response to the fluid shifts experienced in weightlessness. Changes in muscle tone of the vasculature maintain adequate blood flow in spite of the reductions in blood volume during weightlessness. A general cardiac deconditioning, relative to $1 \mathrm{~g}$ status, occurs in flight. Consequently, there are marked alterations in various physiological parameters, such as increased heart rate and mean arterial blood pressure, during exercise.

In addition to functional changes in the heart and vessels of the cardiovascular system, blood components are altered by spaceflight. For instance, red blood cells (RBC) are decreased during spaceflight. This reduction results from either decreased production or increased destruction of the cells. Serum erythropoietin, which stimulates RBC production, is decreased during flight. The increase of serum ferritin in weightlessness indicates increased spleen 


\section{ORGAN/TISSUE SPACE RESEARCH}

breakdown of $\mathrm{RBC}$. The reduction in plasma volume may also contribute to reduced RBC mass. Since $R B C$ are essential for carrying oxygen, this has important implications in getting oxygen to tissues upon return to normal physiological conditions.

A cardiovascular response that may not be directly linked to fluid shifts during spaceflight is increased incidence of dysrhythmias (abnormal heart beats) observed in many crewmembers. While no definitive cause for spaceflight dysrhy thmias has been established, probable factors include gravitational stress (e.g., during reentry), thermal loads (e.g., during extravehicular activity), and electrolyte or hormone alterations.

Significant cardiovascular changes occur as a physiological adaptation to a novel environment. They are not inherently deleterious in microgravity but create the problem of deconditioning upon return to gravitational influence. One of the most significant problems is orthostatic intolerance, the inability to maintain adequate cardiovascular function while standing under the influence of gravity. Postflight tests show increased heart rate and decreased pulse pressure as compared with preflight measurements. Additionally, increased leg volume during orthostatic tests were greater postflight than preflight, suggesting inability to regulate vascular tone following weightlessness. Decrease in exercise capacity is also manifest after spaceflight.

\section{Pulmonary Adaptation to Microgravity}

The pulmonary system exchanges gases with the blood and contributes to the regulation of acid-base balance, which is critical for survival - a sharp shift towards acid or base can be fatal. Anatomically, the respiratory system is comprised of the lungs and the pulmonary circulation. Under the influence of gravity, gradients are established in the lung for gas volume (ventilation) and blood flow (perfusion). These gradients result in greater ventilation and perfusion in the bottom of the lung. The ratio of ventilation to perfusion determines the amount of gas exchange between the air and blood in a given portion of the lung. Therefore, the exchange of gas is best in the lower portions of the lung.

Because of limited data, it is difficult to ascertain the influence of microgravity on the pulmonary system. There have been no reports of postflight abnormalities. Hypothetically, microgravity should alter lung distention, ventilation, and ventilation-perfusion ratios, thus improving gas exchange at rest. Chronic changes in ventilation-perfusion ratios may also affect the function of the right heart. Microgravity effects on maximal oxygen consumption are of the most vital concern because of its potential to limit work capacity in space and upon readaptation to $1 \mathrm{~g}$.

\section{NEUROVESTIBULAR}

\section{Background}

The neurovestibular system controls spatial orientation, coordinated motor performance, and postural maintenance with respect to gravity. Information from specialized organs in the inner ear, along with input from sensory pathways, is integrated in the central nervous system, the brain, to complete these tasks.

The primary structures for obtaining information regarding linear acceleration and the direction of the gravity vector are the otolith organs. These organs contain hair cells embedded in a gelatinous mass containing calcium carbonate crystals called otoconia. Changes in head orientation or linear acceleration impart forces on the otoconia resulting in altered electrical discharge from the hair cells. In addition, the hair cells provide background electrical discharge commensurate with the force of gravity exerted on the otoconia.

The detection of angular acceleration is accomplished by three semicircular canals, accounting for the three planes of orientation. Angular acceleration of the head results in fluid streaming in the semicircular canals corresponding to the plane of movement, ultimately producing an electrical discharge in cells of that canal. Signals from the various sensory organs contribute to the pool of information that is integrated in the brain. This information produces a coordinated signal from the brain to the skeletal and eye muscles. Additionally, there is output to areas of the brain responsible for controlling digestion, blood pressure, and respiration. Pressure sensations induced by gravity alter tactile responses during flight, which may also contribute to postural and spatial orientation alterations.

\section{Adaptation to Spaceflight}

In microgravity, the neurovestibular system must adapt to an altered set of sensory cues, which result in acute changes in the output from, or integration within, the neurovestibular system. Specific adaptations, considered below, include changes in spatial orientation, postural maintenance, the vestibulo-ocular reflex (VOR), and central processing in the neurovestibular system.

Spatial orientation describes the relationship between the body and an external reference frame and is 
accomplished by comparing a variety of external inputs. Spaceflight can lead to impaired integration due to the lack of gravitational effects on the otolith organs. This may result in a sudden reversal of orientation, the so-called inversion illusion. While the semicircular canals are relatively unaffected by microgravity, the detection of static head positions by the otolith organs may be impaired. This alteration may lead to spatial disorientation, resulting in an increased dependence on static visual cues, the use of tactile cues (e.g., from the soles of the feet) to yield an upright sensation, and the alignment of the perceived vertical axis with the long axis of the body.

Maintenance of posture and equilibrium requires integration of information from visual, vestibular, and somatosensory systems. This information coordinates muscular activity to orient the body with respect to gravity. Under gravitational influence, set patterns of muscle activity are the strategy used to adjust automatically the center of gravity to a stable position following perturbations to the body. In microgravity, the sensory interpretation and muscular coordination are changed. These changes are thought to represent altered strategies of response such that the patterns of muscle activity established under gravitational force are changed.

The VOR provides stable vision during head movement. Ocular compensation to head motion is accomplished through a pathway between the semicircular canals and the muscles of the eye. Currently, little is known about the adaptation of the VOR to weightlessness. It is hypothesized that the disparity between sensory input from various sources may result in acute disorientation and motion sickness.

While the adaptations to microgravity render the neurovestibular system well suited for the environment of weightlessness, returning to gravitational influence requires significant readaptation. At present, there is little information describing this process. Alterations in the detection of linear acceleration, a continued increase in the dependence on visual cues and illusions, such as floor motion during vertical movement, occur during the period following weightlessness prior to readaptation. The degree and duration of such symptoms are probably proportional to mission length.

\section{LIVER}

The liver is the "manufacturing plant" of the body producing glucose, blood proteins, and lipids as needed. Additionally, it is the site at which the body removes drugs from the circulatory system. Yet the potential response of the liver to spaceflight is very unclear as there is no useful Earth-bound model that can mimic potential effects on this organ. Some limited data have been obtained from rats flown on Spacelab 3. Undoubtedly, future studies are essential to ascertain whether spaceflight affects the function of this critical organ.

\section{ENDOCRINE ORGANS}

\section{Background}

Many of an organism's adaptations to change in physiological status are related to responses of those that release hormones as part of the endocrine system. These may include the adrenal, pituitary, and thyroid glands, and the pancreas. Hormone balance in the blood controls body metabolism. Response of part of the endocrine system was discussed above in conjunction with the renin-angiotensin-aldosterone response and the change in antidiuretic hormone. Other important hormones include insulin, which promotes fuel storage and maintenance of body protein; cortisol (a glucocorticoid), which promotes the release of fuel from storage; thyroid hormone, which regulates body metabolism by increasing energy production; and growth hormone, which promotes tissue growth and repair. Imbalance of these hormones with altered physiological status has serious implications when the body attempts to respond to stress insults such as injury.

\section{Spaceflight and Simulation Studies}

An increase of blood cortisol occurs in response to stress. Both spaceflight and bedrest simulation increase the amount of cortisol. Another indicator of stress is increased human growth hormone. However, release of growth hormone from the pituitary may be suppressed during spaceflight, as suggested by a study using rat pituitary glands and cells. Indeed, spaceflight may produce some direct effect on growth hormone producing cells in this endocrine gland.

Insulin, which normally counteracts the glucocorticoid (cortisol) action, was found to be diminished after two weeks of spaceflight. Possible loss of this antagonistic effect of insulin could have significant implications for the maintenance of tissue and organ size and the ability to respond to injury in space.

Thyroid hormone, which increases oxygen consumption and heat production, may be increased by spaceflight. This finding is in keeping with elevated oxygen consumption and the high energy demands in space. Coupled with decreased red blood cell mass for transporting oxygen to tissue and organs, there could be serious ramifications of thèse opposing rèspōnsēs. 


\section{ORGAN/TISSUE SPACE RESEARCH}

We have just begun identifying the consequences of space travel on hormone balance. A failure to follow these studies to completion could seriously impair our ability to maintain humans in space for long periods of time. Inflight measurements on humans and animals will be essential because postflight sampling is likely affected by readaptation to gravitational force. Use of animal and human Earth-bound model systems also seems fruitless for these studies as it is not yet possible to mimic the specific hormonal pattern of spaceflight.

\section{BASIC SCIENCE QUESTIONS}

1. What role does gravity play in the development of support structures such as bone and muscle?

2. What is the influence of gravity and its lack on the formation, turnover, and metabolism of support structures?

3. What is the relative importance of altered load bearing and gravitational force in metabolic adaptations of muscle and bone?

4. What is the role of the endocrine system in the response of the support structures to gravitational influence?

5. What role does gravity play in development of bone strength and muscle physiology or the lack of gravity in diminution of these physiological parameters?

6. Is it possible to find a mechanical or electrical perturbation that can substitute for gravity for the development of support structures? If so, are the responses to artificial gravity equivalent to that on Earth?

7. How does gravity influence biomineralization?

8. How does gravity influence physiological systems such as cardiovascular, pulmonary, endocrine, and neurovestibular?

9. To what extent will alteration of organs and tissues in space lead to impaired response? Will any response be pathological, or are they simply adaptive?

10. To what extent are alterations in microgravity reversible upon return to $1 g$ ?

\section{RESEARCH PRIORITIES}

1. Determine the validity of the partially unloaded rat and human bedrest models for predicting spaceflight changes, both short-term and longterm duration, in organs and tissues, especially bone and muscle, and for understanding the basic mechanisms of these changes.

2. Study the dynamic role of calcium in gravitymediated responses of bone.

3. Determine how muscle tension or mechanical strain influence bone growth during skeletal unloading, and whether altered bone growth affects the extent of muscle growth. Such studies should consider interrelation of movement, muscle tension, posture, and skeletal strength.

4. Use the microgravity environment to understand how organisms have adapted their structure to withstand the gravitational influence of Earth during evolution.

5. Determine whether bone crystal size, form, or defect sites are altered by unloading.

6. Determine the mechanism for muscle atrophy and/or altered growth with unloading.

7. Identify the precise pattern of endocrine changes with spaceflight and the ramifications of these changes in terms of organ and tissue functions.

8. Use the microgravity environment to understand how organisms adapted their control (regulation) of organ/tissue function during evolution and how they adapt to changes in their gravitational environment.

9. Dissect the possible components of the gravitational influence to evaluate which is the major contributing factor in each adaptive response.

\section{BIBLIOGRAPHY}

Committee on Space Biology and Medicine. 1987. A Strategy for Space Biology and Medical Science for the 1980s and 1990s (The Goldberg Report). Washington, DC: National Academy Press, 220 p.

Desplanches, D., Mayet, M.H., Ilyina-Kakueva, E.I., Sempore, B., and Flandrois, R. 1990. Skeletal 


\section{TISCHLER AND MOREY-HOLTON}

muscle adaptation in rats flown in Cosmos 1667. Journal of Applied Physiology 68(1): 48-52.

Grindeland, R., Hymer, W.C., Farrington, M., Fast, T., Hayes, C., Motter, K., Patil, L., and Vasques, M. 1987. Changes in pituitary growth hormone cells prepared from rats flown on Spacelab 3. American Journal of Physiology 252(2, Part 2): R209-R215.

Grindeland, R.E., Ed. 1990. Cosmos 1887. FASEB Journal 4(1): 10-109.

Holy, X. and Mounier, Y. 1991. Effects of short spaceflights on mechanical characteristics of rat muscles. Muscle \& Nerve 14(1): 70-78.

Martin, T.P. 1988. Protein and collagen content of rat skeletal muscle following space flight. Cell and Tissue Research 254(1): 251-253.

Merrill, A.H., Wang, E., Jones, D.P., and Hargrove, J.L. 1987. Hepatic function in rats after spaceflight: effects on lipids, glycogen, and enzymes. American Journal of Physiology 252(2, Part 2): R222-R226.

Morey, E.R. and Baylink, D.J. 1978. Inhibition of bone formation during space flight. Science 201(4361): 1138-1141.

Morey-Holton, E. and Tischler, M. (Eds.) 1988. NASA Workshop on Biological Adaptation. Moffett Field, CA: NASA, Ames Research Center, 102 p. (NASA TM-89468)

Morey-Holton, E.R. and Arnaud, S.B. 1991. Skeletal responses to spaceflight. In: Advances in Space Biology and Medicine, Vol. 1. (Bonting, S.L., Ed.). Greenwich, CT: JAI Press, p. 37-69.

Musacchia, X.J. and Steffen, J.M. 1984. Cardiovascular and hormonal (aldosterone) responses in a rat model which mimics response to weightlessness. Physiologist 27(6): S41-S42.

Musacchia, X.J. and Steffen, J.M. 1982. Short term ( 1 and 3 day) cardiovascular adjustments to suspension antiorthostasis in rats. Physiologist 25(6): $\$ 163$ S164.
Musacchia, X.J., Steffen, J.M., Fell, R.D., and Dombrowski, M.J. 1990. Skeletal muscle response to spaceflight, suspension, and recovery in rats. Journal of Applied Physiology 69(6): 22482253.

Nicogossian, A.E., Huntoon, C.L., and Pool, S.L. (Eds.) 1989. Space Physiology and Medicine, 2nd Edition. Philadelphia: Lea \& Febiger, 421 p.

Oganov, V.S., Rakhmanov, A.S., Novikov, V.E., et al. 1991. The state of human bone tissue during space flight. Acta Astronautica 23: 129-133.

Riley, D.A., Ellis, S., Slocum, G.R., Satyanarayana, T., Bain, J.L.W., and Sedlak, F.R. 1987. Hypogravity-induced atrophy of rat soleus and extensor digitorum longus muscles. Muscle \& Nerve 10: 560-568.

Sandler, H. and Vernikos, J. (Eds.) 1986. Inactivity: Physiological Effects. Orlando, FL: Academic Press, 205 p.

Steffen, J.M. and Musacchia, X.J. 1986. Spaceflight effects on adult rat muscle protein, nucleic acids, and amino acids. American Journal of Physiology 251: R1059-R1063.

Task Group on Life Sciences. 1988. Space Science in the Twenty-First Century: Imperatives for 1995-2015 Life Sciences. Washington, DC: National Academy Press.

Tischler, M.E., Jaspers, S.R., Henriksen, E.J., and Jacobs, S. 1985. Responses of skeletal muscle to unloading - A review. Physiologist 28(6): S13S16.

Wronski, T.J. and Morey, E.R. 1983. Alterations in calcium homeostasis and bone during actual and simulated space flight. Medicine and Science in Sports and Exercise 15(5): 410-414.

Wronski, T.J and Morey, E.R. 1983. Effect of spaceflight on periosteal bone formation in rats. American Journal of Physiology 244(3): R305R309. 